\title{
Factors involved in ubiquitination and deubiquitination of PEX5, the peroxisomal shuttling receptor
}

Tony A. Rodrigues ${ }^{a, b}$, Tânia Francisco ${ }^{a, b}$, Andreia F. Carvalho a , Manuel P. Pinto a, Cláudia P. Grou ${ }^{a}$, and Jorge E. Azevedo ${ }^{a, b, *}$

aOrganelle Biogenesis and Function Group, Instituto de Biologia Celular e Molecular (IBMC), Universidade do Porto, R. do Campo Alegre, 823, 4150-180 Porto, Portugal, and 'Instituto de Ciências Biomédicas Abel Salazar (ICBAS), Universidade do Porto, R. de Jorge Viterbo Ferreira, 228, 4050-313 Porto, Portugal.

${ }^{*}$ Corresponding author: Jorge. E. Azevedo, Organelle Biogenesis and Function Group, Instituto de Biologia Celular e Molecular (IBMC), Universidade do Porto, R. do Campo Alegre, 823, 4150-180 Porto, Portugal; Tel.: (+351) 226074900; Fax: (+351) 226099157; e-mail: jazevedo@ibmc.up.pt.

\section{Summary}

Peroxisomal matrix proteins are synthesized on cytosolic ribosomes and posttranslationally targeted to the organelle by the soluble factor PEX5. Besides a role as a receptor, and probably as a chaperone, PEX5 also holds the key to the matrix of the organelle. Indeed, the available data suggest that PEX5 itself pushes these proteins across the peroxisomal membrane using as driving force the strong protein-protein interactions that it establishes with components of the peroxisomal membrane docking/translocation module (DTM). In recent years, much has been learned on how this transport system is reset and kept fine-tuned. Notably, this involves covalent modification of PEX5 with ubiquitin. Two types of PEX5 ubiquitination have been characterized: monoubiquitination at a conserved cysteine, a mandatory event for the extraction of PEX5 from the DTM; and polyubiquitination, probably the result of a quality control mechanism aiming at clearing the DTM from entangled PEX5 molecules. Monoubiquitination of PEX5 is transient in nature and the factors that reverse this modification have recently been identified.

\section{Keywords}

Peroxisome, PEX5, protein trafficking, ubiquitination.

\section{Abbreviations}

AAA, ATPases associated with diverse cellular activities; DTM, Docking/Translocation Module; DUB, deubiquitinase; E1, ubiquitin-activating enzyme; E2, ubiquitin-conjugating enzyme; E3, ubiquitin ligase; PEX, peroxin; PIM, Peroxisomal Import Machinery; PTS, peroxisome targeting signal; REM, Receptor Export Module; RING, Really Interesting New Gene; TPRs, tetratricopeptide repeats; Ub, ubiquitin. 


\section{Introduction}

Peroxisomes are single membrane-bound organelles involved in numerous metabolic pathways ( $\mathrm{Hu}$ et al. 2012; Islinger et al. 2010; Michels et al. 2006; Wanders and Waterham 2006). Their importance for human health and development is dramatically illustrated by a group of genetic diseases, the peroxisomal biogenesis disorders, in which peroxisome functions are partially or even completely impaired (Waterham and Ebberink 2012). Peroxisomal biogenesis disorders are caused by mutations in PEX genes, which encode proteins specifically involved in peroxisome maintenance and inheritance, the socalled peroxins (Distel et al. 1996). The majority of mammalian peroxins are components of the peroxisomal protein import machinery (PIM) (Fig. 4.4.1). Collectively these proteins ensure the correct delivery of newly synthesized proteins to the organelle lumen. Most PIM peroxins are part of one of two functional/structural units: PEX13, PEX14, and the "Really Interesting New Gene" (RING) peroxins PEX2, PEX10, and PEX12 compose the membrane-embedded docking/translocation module (DTM; Agne et al. 2003; Oeljeklaus et al. 2012; Reguenga et al. 2001); the two peroxisomal "ATPases associated with diverse cellular activities" (AAA ATPases), PEX1 and PEX6, together with their peroxisomal membrane anchor, PEX26, comprise the receptor export module (REM; Matsumoto et al. 2003; Birschmann et al. 2003). The peroxisomal protein shuttling receptor PEX5 and its adaptor protein PEX7 complete the list of mammalian peroxins that integrate the PIM (Dodt et al. 1995; Fransen et al. 1995; Braverman et al. 1997). In addition to peroxins, the mammalian PIM also comprises other proteins, which are mostly involved in ubiquitination/deubiquitination events. Due to the fact that their function is not restricted to the PIM they are not classified as peroxins.

The mammalian peroxins referred to above have orthologs in all peroxisomecontaining organisms characterized so far, from yeasts and fungi to plants (Kiel et al. 2006; Schluter et al. 2006). Strikingly, however, the reverse is not true. Indeed, several peroxins found in plants and lower eukaryotes do not exist in mammals. Apparently, evolution led to simpler PIMs. At least two different mechanisms seem to be behind this simplification. In one case, the function of two yeast/fungi/plant peroxins, PEX4 and PEX22, ended up being carried out by a family of mammalian ubiquitin-conjugating enzymes (E2D1/2/3) involved in many other pathways. In another case, a peroxin (PEX5) acquired the capacity to perform two different tasks (import of both PTS1- and PTS2containing proteins; see below section 2.1) (Braverman et al. 1998; Galland et al. 2007; Otera et al. 1998; Woodward and Bartel 2005), each of which is performed by a different peroxin in yeasts/fungi (see Schliebs and Kunau 2006, and references cited therein). Despite these differences, the basics of the mechanism of protein import into the peroxisomal matrix remained relatively well conserved during evolution (Galland and Michels 2010; Grou et al. 2009a; Hu et al. 2012; Platta et al. 2013; Ma and Subramani 2009).

\section{The PEX5-mediated peroxisomal matrix protein import pathway}

The import pathway of newly synthesized proteins into the matrix of the organelle is generally described using a PEX5-centered perspective and can be divided into two parts (see Fig. 4.4.2). The first part comprises three steps, all independent of cytosolic ATP, 
and culminates with the translocation of the cargo protein across the peroxisomal membrane, as briefly explained below. The second part, on the other hand, includes the energy-requiring steps necessary to reset the protein transportation system. As we shall see, ubiquitin plays a major role here.

\subsection{The ATP-independent steps}

Peroxisomal matrix proteins possess one of two types of peroxisomal targeting signals (PTSs). The majority of them harbor a PTS type 1 (PTS1), a tripeptide with the sequence S-K-L or a variant present at their extreme C-termini (Brocard and Hartig 2006; Gould et al. 1989). A few peroxisomal matrix proteins possess instead a PTS2. This is an $\mathrm{N}$-terminal degenerated nonapeptide with the sequence $(\mathrm{R} / \mathrm{K})-(\mathrm{L} / \mathrm{V} / \mathrm{I})-\mathrm{X}_{5}-(\mathrm{H} / \mathrm{Q})-(\mathrm{L} / \mathrm{A})$ (Kunze et al. 2011; Lazarow 2006; Swinkels et al. 1991). In contrast to the PTS1, which remains intact upon import, the PTS2 is generally cleaved in the peroxisomal matrix by a serine protease (Kurochkin et al. 2007; Schuhmann et al. 2008). In mammals, plants and many other organisms, all peroxisomal matrix proteins are transported to the peroxisome by PEX5 (Otera et al. 1998; Braverman et al. 1998; Galland et al. 2007; Woodward and Bartel 2005). This is a 70-kDa monomeric protein which in vivo displays a dual subcellular localization, peroxisomal and cytosolic, reflecting its role as a shuttling receptor (CostaRodrigues et al. 2005; Dodt et al. 1996; Shiozawa et al. 2009). Interestingly, binding of PEX5 to newly synthesized matrix proteins that are oligomeric in their native state strongly inhibits their oligomerization, suggesting that PEX5 is also a chaperone/holdase (Freitas et al. 2011). This property is probably crucial to avoid premature oligomerization in the cytosol of proteins that no longer expose their PTS1 upon oligomerization (Luo et al. 2008; Tanaka et al. 2008). Structurally, PEX5 comprises two main domains: 1) a natively unfolded N-terminal half that interacts with the peroxins PEX7, PEX13, and PEX14 (Braverman et al. 1998; Carvalho et al. 2006; Costa-Rodrigues et al. 2005; Otera et al. 2000; Saidowsky et al. 2001); and 2) a structured C-terminal half possessing seven tetratricopeptide repeats (TPRs) domains (Gatto et al. 2000). The interaction between PEX5 and PTS1 proteins is direct and sufficient to ensure that these proteins are efficiently targeted to the organelle. The interaction involves the PTS1 peptide on one side, and the TPR domains of PEX5 on the other, but the N-terminal half of PEX5 also contributes for the interaction (Braverman et al. 1998; Freitas et al. 2011; Gunkel et al. 2004; Klein et al. 2001; Klein et al. 2002; Oshima et al. 2008). The PTS2-PEX5 interaction requires the adaptor protein PEX7 (Galland et al. 2007; Lazarow 2006; Otera et al. 1998; Woodward and Bartel 2005; Braverman et al. 1998). In lower eukaryotes, PEX5 does not interact with PEX7, and therefore the receptor function of PEX5 is restricted to PTS1 proteins. In these organisms, targeting of PTS2 proteins is ensured by a species-specific receptor (PEX20, PEX18 or PEX21) which displays structural/functional similarities with the N-terminal half of mammalian PEX5, including the capacity to interact with PEX7 (Dodt et al. 2001; Lazarow 2006; Schliebs and Kunau 2006; Einwachter et al. 2001). We refer to these peroxins as PEX5-like proteins.

Following cargo recognition, PEX5 interacts with the DTM in a reversible manner; this docking step is then followed by the temperature-dependent insertion of PEX5 into the DTM (Costa-Rodrigues et al. 2004; unpublished results). PEX5 at this stage displays 
a transmembrane topology having most of its polypeptide chain facing the peroxisomal matrix, whereas a 2-kDa N-terminal portion remains exposed to the cytosol (Gouveia et al. 2003a; Gouveia et al. 2000). Importantly, insertion of PEX5 into the DTM is a cargo protein-dependent process (Gouveia et al. 2003b). These observations are at the basis of the current model proposing that PEX5 pushes cargo proteins across the peroxisomal membrane as it gets inserted into the DTM (Azevedo et al. 2004; Grou et al. 2009a; Oliveira et al. 2003). Remarkably, in vitro import experiments have shown that neither insertion of PEX5 into the DTM nor translocation of cargo proteins across the peroxisomal membrane are affected by non-hydrolyzable ATP analogs or by ATP depletion of the import assays (Alencastre et al. 2009; Oliveira et al. 2003; unpublished results). Likewise, ionophores have no effect on any of these events (Alencastre et al. 2009), in agreement with the fact that the peroxisomal membrane is readily permeable to small ions/molecules (Antonenkov and Hiltunen 2012; Rokka et al. 2009). Apparently, the PIM uses neither the energy of ATP hydrolysis nor a membrane potential to transport proteins from the cytosol into the organelle matrix. Altogether, these findings led us to propose that the driving force for the cargo protein translocation step resides in the strong protein-protein interactions that PEX5 establishes with components of the DTM (Oliveira et al. 2003).

\subsection{Resetting the peroxisomal protein import machinery}

The interaction of DTM-embedded PEX5 with components of this membrane module is essentially irreversible (Costa-Rodrigues et al. 2004). Therefore, it is not surprising that extraction of the receptor back into the cytosol requires energy input. Understanding the details of this extraction step was a difficult but particularly interesting task because it ended up revealing that the DTM is also an ubiquitin ligase. Indeed, PEX5 is monoubiquitinated every time it passes through the DTM, a mandatory modification for its subsequent ATP-dependent extraction back into the cytosol.

\subsubsection{PEX5 monoubiquitination}

As stated above, DTM-embedded PEX5 exposes approximately $2 \mathrm{kDa}$ of its $\mathrm{N}$ terminus to the cytosol. This small $\mathrm{N}$-terminal domain includes a cysteine-containing motif that is conserved not only in PEX5 proteins from all organisms, but also in PEX5-like proteins. Interestingly, deletion of the first 17 amino acids of human PEX5, which contains this motif, does not affect its capacity to get inserted into the DTM in a cargo-dependent manner, but renders it completely incompetent in the export step (Costa-Rodrigues et al. 2004). Likewise, deletion of the first $19 \mathrm{~N}$-terminal amino acids of Pichia pastoris PEX20 also interferes with the normal recycling step (Leon et al. 2006). Similar results were observed when the conserved cysteine of both P. pastoris PEX20 and human PEX5 was replaced by a serine (Carvalho et al. 2007a; Leon and Subramani 2007). Clearly, this conserved cysteine residue has a determinant role in the receptor recycling step but the reason why it is so important became apparent only when this residue was found to be monoubiquitinated (Carvalho et al. 2007b; Williams et al. 2007). This modification is absolutely required for the next step of the PEX5-mediated protein import pathway, the extraction of monoubiquitinated PEX5 back into the cytosol (Carvalho et al. 2007b; Platta et al. 2007). Recent data confirmed that the PEX5-like proteins, PEX20 and PEX18, are 
also ubiquitinated at the conserved cysteine residue (Hensel et al. 2011; Liu and Subramani 2013).

The reason why a cysteine, and not the classical lysine residue, is the acceptor of ubiquitin in this reaction remains unknown. This is even more puzzling when we take into consideration the fact that substitution of the conserved cysteine residue in PEX5 by a lysine results in a seemingly normal protein that enters the DTM, receives a single ubiquitin molecule and is exported back into the cytosol as efficiently as the wild type protein in in vitro assays. Furthermore, when expressed in embryonic fibroblasts from a PEX5 knockout mouse, this PEX5 mutant protein is capable of restoring peroxisomal protein import, again, as efficiently as the wild type PEX5 protein (Grou et al. 2009b). Nevertheless, some hypotheses regarding the conserved cysteine have been formulated. These include the possibility to deubiquitinate cytosolic Ub-PEX5 using a non-enzymatic mechanism (see below section 2.2.4) or the potential to block the DTM under some conditions (e.g., oxidative stress) through chemical modification of the conserved cysteine residue (e.g., oxidation, glutathiolation, nitrosylation, etc.). Obstruction of the DTM by export-incompetent PEX5 molecules would result in a cytosolic localization for newly synthesized peroxisomal enzymes (e.g., catalase, epoxide hydrolase and glutathione Stransferase $\mathrm{k}$ ), a situation that might be advantageous under some stress conditions (see also Fransen et al. 2012 and Grou et al. 2009b).

\subsubsection{PEX5 dislocation}

It is presently believed that monoubiquitination of DTM-embedded PEX5 serves no purpose other than preparing the receptor for the export step. Several arguments support this idea. First, as stated above, insertion of cargo-loaded PEX5 into the DTM in an in vitro import system is not affected when ATP is removed from the assays (Oliveira et al. 2003). Under these conditions, monoubiquitination of PEX5 at the DTM is no longer possible because the ubiquitin-activating enzyme (E1) uses ATP to activate ubiquitin. Likewise, PEX5 proteins lacking the conserved cysteine are still able to enter the DTM where they acquire the expected transmembrane topology (Carvalho et al. 2007a; Carvalho et al. 2007b). Furthermore, and in agreement with these findings, PEX5mediated import of pre-thiolase, a PTS2 protein, and its processing in the peroxisomal matrix are also not affected by removal of ATP from import reactions and similar results were obtained recently in our laboratory when studying the import pathway of a PTS1 protein (Alencastre et al. 2009; unpublished results). Thus, monoubiquitination is necessary neither for the docking/insertion steps of PEX5 into the DTM nor for cargo protein translocation and release into the peroxisomal matrix. On the other hand, as stated above, PEX5 mutant proteins that cannot be monoubiquitinated are not substrates for the REM and accumulate at the DTM. Furthermore, monoubiquitination of PEX5 in in vitro import assays using a GST-ubiquitin fusion protein leads to the same outcome (Carvalho et al. 2007b). Altogether, these findings suggest that it is not the covalent modification of PEX5 per se that prepares the receptor for the export step (e.g., by inducing a conformational alteration of PEX5), but rather that the ubiquitin moiety in the DTM-embedded Ub-PEX5 conjugate provides a context-specific "handle" for the REM. 
How the REM peroxins, PEX1 and PEX6, recognize DTM-embedded Ub-PEX5 remains largely unknown. Nevertheless, recent data suggest that the interaction between Ub-PEX5 and the REM may not be direct (Miyata et al. 2012). Indeed, using a mammalian in vitro import/export assay the authors found a cytosolic protein that stimulated export of PEX5 from the DTM. The protein was identified as AWP1, an ubiquitin-binding protein best known for its participation in the NF-kB signaling pathway (Chang et al. 2011). Further biochemical characterization of this protein led the authors to propose that AWP1 mediates the interaction between monoubiquitinated PEX5 and the REM (Miyata et al. 2012).

\subsubsection{PEX5 deubiquitination}

Export of monoubiquitinated PEX5 from the DTM can be easily observed using a mammalian peroxisomal in vitro assay, particularly if the export reaction is made in the presence of a general deubiquitinase (DUB) inhibitor (e.g., ubiquitin aldehyde) (Grou et al. 2009b). In contrast, all attempts to detect the mammalian or yeast Ub-PEX5 thioester conjugate in cytosolic fractions obtained from cells/organs yielded negative results; UbPEX5 could only be detected in organelle fractions (Grou et al. 2009b; Williams et al. 2007). Apparently, in vivo Ub-PEX5 is deubiquitinated very rapidly after export from the DTM. The factors that catalyze this deubiquitination step have been recently identified. Using biochemical approaches, UBP15 in Saccharomyces cerevisiae and USP9X in mammals have been identified as the DUBs acting on Ub-PEX5. Interestingly, however, knockout and knockdown of UBP15 and USP9X genes, respectively, did not result in the cytosolic accumulation of Ub-PEX5 (Debelyy et al. 2011; Grou et al. 2012). Obviously, there are other ways to deubiquitinate PEX5. These may include other less specific/active DUBs (Debelyy et al. 2011; Grou et al. 2012) or, as proposed previously, even a nonenzymatic mechanism because the thioester bond linking ubiquitin to PEX5 is much more labile than the typical isopeptide bond found in most ubiquitin conjugates (Grou et al. 2009b). Indeed, soluble Ub-PEX5 (but not DTM-embedded Ub-PEX5) is easily disrupted in the presence of $5 \mathrm{mM}$ glutathione (a physiological concentration) displaying a half-life of just $2.3 \mathrm{~min}$ (Grou et al. 2009b).

\subsubsection{The ubiquitin-conjugating enzymes (E2s) and the ubiquitin ligase(s) (E3) of the PEX5-mediated protein import pathway}

In order to react with a protein, ubiquitin has first to be activated by the ATPdependent ubiquitin-activating enzyme (E1) yielding a thioester intermediate. This activated form of ubiquitin is then transferred to the catalytic cysteine of an ubiquitinconjugating enzyme (E2). Finally, the Ub-E2 thioester conjugate is recruited by an ubiquitin ligase $(E 3)$ to the vicinity of the protein substrate which will then react with the activated ubiquitin (Spasser and Brik 2012). The specificity of a given protein ubiquitination reaction is thus imposed by the particular E2/E3 pair that catalyses that reaction (Metzger et al. 2013).

Most eukaryotic organisms have 1-2 E1s, dozens of E2s and an even larger number of E3s (e.g., more than 600 in mammals) (Hutchins et al. 2013). The latter can be grouped into several different classes (Metzger et al. 2012), but of relevance here are the 
RING family of ubiquitin ligases (Deshaies and Joazeiro 2009). These E3s are characterized by a small protein domain (the RING domain) that binds two $\mathrm{Zn}^{2+}$ ions and adopts a typical cross-braced folding (Budhidarmo et al. 2012). RING domains have two distinct functions. First, they interact directly with E2s acting as recruitment platforms for $\mathrm{Ub}$-E2 conjugates; second, they increase the reactivity of the E2-bound ubiquitin by repositioning the ubiquitin molecule further exposing the thioester bond to nucleophile attack (Metzger et al. 2013). This activation phenomenon is frequently explored in in vitro ubiquitination assays to test whether a RING domain really has E3 activity and also to define the E2(s) that are accepted by a given E3. Several experimental readouts are used in these assays, including monitoring auto-ubiquitination of the E3, ubiquitination of model substrates or even E3-dependent reactivity of the E2-Ub conjugate with single amino acids (e.g., lysine and cysteine) (Wenzel et al. 2011; Swanson et al. 2001). In many of these assays, proximity of the nucleophilic protein/amino acid substrate to the RING-E2$\mathrm{Ub}$, more than its identity, is the crucial factor. This is achieved either by simply increasing the concentration of the substrate or by fusing it to the RING domain.

E3s can also be classified according to the mechanisms they use to recruit substrates. Some E3s possess substrate recruiting domains in other regions of their polypeptide chain (Metzger et al. 2013). Many, however, are subunits of larger proteins complexes and depend on other partners of the complex to recruit the substrate (e.g., Cullin RING ligases; Sarikas et al. 2011). These are frequently referred to as multi-subunit E3s (see also Metzger et al. 2013).

Which E2s and E3s participate in the PEX5-mediated protein import pathway? The first answer to this question was provided by two independent studies on yeast PEX4, an E2 long-known for its involvement in peroxisomal protein import (Wiebel and Kunau 1992). Indeed, it was shown that yeast strains lacking PEX4 do not monoubiquitinate PEX5 at the conserved cysteine residue (Williams et al. 2007), and are unable to recycle peroxisomal PEX5 back into the cytosol (Platta et al. 2007). Interestingly, and in contrast to the majority of E2s which are soluble proteins, PEX4 is stable and active only when bound to the peroxisomal membrane by another protein, PEX22 (Koller et al. 1999). Orthologs of both PEX4 and PEX22 have been found using bioinformatic analyses in several yeasts/fungi and plants, suggesting that all these organisms have a peroxisomal E2 dedicated to the peroxisomal protein import pathway (Kiel et al. 2006; Schluter et al. 2006; Zolman et al. 2005). Strikingly, however, no orthologs could be found in mammals and many other organisms. Proteomic studies aiming at identifying new mammalian peroxisomal proteins also failed to reveal the existence of a peroxisome-bound E2 (Islinger et al. 2007; Kikuchi et al. 2004; Wiese et al. 2007). An explanation for these negative findings was provided by a biochemical characterization of the mammalian E2. Using an in vitro system that recapitulates all steps of the peroxisomal protein import pathway, it was found that a low-speed centrifugation of post-nuclear supernatants was sufficient to separate peroxisomes from the E2 activity involved in PEX5 monoubiquitination. Purification of this activity led to the identification of three almost identical cytosolic E2s, E2D1/2/3 (UbcH5a/b/c in humans) (Grou et al. 2008), a group of E2s involved in many other biological pathways (Gonen et al. 1999; Saville et al. 2004). 
As stated above, monoubiquitination of PEX5 at the conserved cysteine occurs at the DTM after cargo protein-dependent insertion of the receptor into this membrane module. Importantly, monoubiquitination of PEX5 can also be observed in an in vitro system comprising highly pure rat liver peroxisomes and recombinant $\mathrm{E} 1$ and $\mathrm{UbcH} 5 \mathrm{c}$ (Grou et al. 2008), a finding strongly suggesting that the DTM itself is the E3 ligase catalyzing this unconventional ubiquitination. Three of the five core components of this module are the RING peroxins, PEX2, PEX10 and PEX12 and thus they are the most obvious candidates to perform this function. Interestingly, several studies suggest that PEX5 can still enter the DTM in cells lacking RING peroxins (Agne et al. 2003; Chang et al. 1999; Collins et al. 2000; Dodt and Gould 1996). Apparently, and similarly to multi-subunit E3s, the substraterecruiting function of the DTM/E3 resides not in the RING peroxins but rather in other subunits of the complex.

Detailed mechanistic data on how the DTM monoubiquitinates PEX5 are still scarce. In vitro ubiquitination assays using recombinant RING domains from yeast and plant peroxins have shown that they all display E3 activity when assayed with human UbcH5 (Kaur et al. 2013; Williams et al. 2008) or with yeast PEX4 (Platta et al. 2009). Thus, in principle, any of the three RING peroxins could promote monoubiquitination of PEX5 at the conserved cysteine. However, data supporting this possibility are not yet available, despite some attempts (Platta et al. 2009). A definite answer to this question will probably require reconstituting a major part of the DTM/E3 using purified components and determine which of the RING peroxins, if any individually, is capable of promoting the correct type of ubiquitination, at the correct amino acid residue of PEX5. Such experiments may turn out to be quite demanding specially if we take into consideration very recent in vivo data suggesting that RING peroxins are not redundant and that all three together are required for receptor mono- and polyubiquitination (Liu and Subramani 2013, and see below section 3).

\section{Polyubiquitination of PEX5}

Monoubiquitination of PEX5 is not the only type of ubiquitination occurring at the DTM. In yeast mutant strains lacking PIM components that act at late steps of the pathway (i.e., PEX5 monoubiquitination and its ATP-dependent dislocation from the DTM), small amounts of polyubiquitinated PEX5 are detected in peroxisomes (Kiel et al. 2005a; Platta et al. 2004; Kragt et al. 2005). Furthermore, the steady-state levels of PEX5 are diminished in some of these mutant strains (Collins et al. 2000; Koller et al. 1999) suggesting that this polyubiquitination event targets PEX5 for proteasomal degradation. A similar decrease in the steady-state levels of PEX5 in human cell lines from some patients with Peroxisome Biogenesis Disorders was also reported (Yahraus et al. 1996; Dodt and Gould 1996). Further characterization of this phenomenon in yeasts revealed that polyubiquitination of PEX5 is mediated by the multipurpose E2s Ubc1/Ubc4/Ubc5 (Kiel et al. 2005a; Kragt et al. 2005; Platta et al. 2004). Polyubiquitination of PEX5 does not seem to occur at the conserved cysteine residue; rather, one or two lysines located near the conserved cysteine have been identified as the ubiquitination sites (Kiel et al. 2005b; Williams et al. 2007). Although polyubiquitination of PEX5 has not yet been detected in wild type strains, and substitution of those two PEX5 lysines by arginines has no 
phenotypic effects (Platta et al. 2007), it is possible, nevertheless, that this alternative way to remove PEX5 from the DTM is important whenever the normal recycling mechanism cannot be used, e.g. if PEX5 becomes entangled in the DTM.

\section{Conclusions}

The first clue that ubiquitin should play some role in the peroxisomal protein import pathway dates back to 1992 when one of the yeast genes involved in this pathway was found to encode the ubiquitin-conjugating enzyme, PEX4 (Wiebel and Kunau 1992). The awareness, a few years later, that the three RING peroxins present in all peroxisomecontaining organisms might well be members of a vast family of ubiquitin ligases E3s (Joazeiro and Weissman 2000), fed this suspicion. However, the main mechanistic connection between ubiquitin and the PIM remained elusive for many years, and only in 2007 did we understand that ubiquitination at the PIM is not simply a manifestation of the quality control ubiquitin-proteasome system. Rather, ubiquitination is a mandatory step of this protein sorting pathway, occurring every single time a PEX5 molecule delivers a cargo protein into the matrix of the organelle. Clearly, the disruption of the Ub-PEX5 thioester conjugate by the thiol reagents commonly used in SDS-PAGE analyses tricked many researchers in the field, us included, for too long.

Any new finding ends up raising more questions than those it solved. One of the numerous questions still waiting for an answer is why a cysteine and not a lysine residue is used as the ubiquitin acceptor in PEX5. Also, the enzymology of the RING peroxins remains vastly unknown. How do they function? How are they regulated? In principle, either a single RING domain or a dimer of RINGs (Metzger et al. 2013) should be sufficient to catalyze monoubiquitination of PEX5. Why then are the three RING peroxins non-redundant and all necessary for receptor monoubiquitination (Liu and Subramani 2013)? Is it possible that the three RING peroxins are actually modules of a multi-RING E3 ligase? Obviously there are still many other new findings to be made.

\section{FIGURE LEGENDS}

Fig. 4.4.1. Components of the peroxisomal protein import machinery (PIM).

The components of the PIM are organized into structural/functional units. The Docking/Translocation Module (DTM), a membrane-embedded protein complex comprises: PEX13, PEX14, and the RING peroxins PEX2, PEX10, and PEX12, whereas PEX1 and PEX6 and their membrane-anchor, PEX26, constitute the Receptor Export Module (REM). The Shuttling Receptor (R), a cargo protein (CP), ubiquitin (Ub), AWP1 (a soluble protein involved in receptor recycling), and the ubiquitin-conjugating enzyme E2 are also depicted. In some organisms the E2 is bound to the peroxisomal membrane via a membrane anchor (dashed-line shape).

\section{Fig. 4.4.2. The PEX5-mediated protein import pathway.}

The PEX5-mediated protein import pathway comprises eight steps. The PIM intermediates are referred to as stages, which are numbered 0 to 4 (some substages "a" and "b" are of conceptual nature). PEX5 (stage 0) binds a cargo protein (CP) in the 
cytosol (stage 1a). It then docks at the Docking/Translocation Module (DTM; stage 1b), where it gets inserted with the concomitant translocation of the cargo protein into the organelle matrix (stage 2). PEX5 is then monoubiquitinated (stage 3a), so that it can be recognized by the Receptor Export Module (REM) and dislocated into the cytosol, in an ATP-dependent manner (stage $3 b$ to stage 4b). Finally, cytosolic Ub-PEX5 is deubiquitinated probably by a combination of enzymatic (DUBs) and non-enzymatic (GSH) mechanisms regenerating free PEX5 (stage 0). Strategies/reagents that have been used to block this pathway at different steps are indicated $(\otimes)$. Note that ATPYS, a nonhydrolyzable ATP analogue is efficiently used by the ubiquitin-activating enzyme (E1), but not by the REM. Ub, ubiquitin; E2, ubiquitin-conjugating enzyme; DUBs, deubiquitinases; GSH, glutathione; GST-Ub, GST-tagged ubiquitin; cyt, cytosol; mat, peroxisomal matrix.

\section{Acknowledgements}

This work was funded by FEDER funds through the Operational Competitiveness Programme - COMPETE and by National Funds through FCT - Fundação para a Ciência e a Tecnologia under the project FCOMP-01-0124-FEDER-019731 (PTDC/BIABCM/118577/2010). T. A. R., T. F., M. P. P. and C. P. G. are supported by Fundação para a Ciência e a Tecnologia, Programa Operacional Potencial Humano do QREN, and

Fundo Social Europeu. A. F. C. is supported by Programa Ciência, funded by Programa Operacional Potencial Humano do QREN, Tipologia 4.2, Promoção do Emprego Científico, by Fundo Social Europeu and by national funds from Ministério da Ciência, Tecnologia e Ensino Superior.

\section{References}

Agne B, Meindl NM, Niederhoff K, Einwachter H, Rehling P, Sickmann A, Meyer HE, Girzalsky W, Kunau WH (2003) Pex8p: an intraperoxisomal organizer of the peroxisomal import machinery. Mol. Cell 11 (3):635-646.

Alencastre IS, Rodrigues TA, Grou CP, Fransen M, Sa-Miranda C, Azevedo JE (2009) Mapping the cargo protein membrane translocation step into the PEX5 cycling pathway. J. Biol. Chem. 284 (40):27243-27251.

Antonenkov VD, Hiltunen JK (2012) Transfer of metabolites across the peroxisomal membrane. Biochim. Biophys. Acta 1822 (9):1374-1386.

Azevedo JE, Costa-Rodrigues J, Guimaraes CP, Oliveira ME, Sa-Miranda C (2004) Protein translocation across the peroxisomal membrane. Cell Biochem. Biophys. 41 (3):451-468.

Birschmann I, Stroobants AK, van den Berg M, Schafer A, Rosenkranz K, Kunau WH, Tabak HF (2003) Pex15p of Saccharomyces cerevisiae provides a molecular basis for recruitment of the AAA peroxin Pex6p to peroxisomal membranes. Mol. Biol. Cell 14 (6):2226-2236.

Braverman N, Dodt G, Gould SJ, Valle D (1998) An isoform of pex5p, the human PTS1 receptor, is required for the import of PTS2 proteins into peroxisomes. Hum. Mol. Genet. 7 (8):1195-1205. 
Braverman N, Steel G, Obie C, Moser A, Moser H, Gould SJ, Valle D (1997) Human PEX7 encodes the peroxisomal PTS2 receptor and is responsible for rhizomelic chondrodysplasia punctata. Nat Genet 15 (4):369-376.

Brocard C, Hartig A (2006) Peroxisome targeting signal 1: is it really a simple tripeptide? Biochim. Biophys. Acta 1763 (12):1565-1573.

Budhidarmo R, Nakatani Y, Day CL (2012) RINGs hold the key to ubiquitin transfer. Trends Biochem. Sci. 37 (2):58-65.

Carvalho AF, Costa-Rodrigues J, Correia I, Costa Pessoa J, Faria TQ, Martins CL, Fransen M, Sa-Miranda C, Azevedo JE (2006) The N-terminal half of the peroxisomal cycling receptor Pex5p is a natively unfolded domain. J. Mol. Biol. 356 (4):864-875.

Carvalho AF, Grou CP, Pinto MP, Alencastre IS, Costa-Rodrigues J, Fransen M, SaMiranda C, Azevedo JE (2007a) Functional characterization of two missense mutations in Pex5p - C11S and N526K. Biochim. Biophys. Acta 1773 (7):1141-1148.

Carvalho AF, Pinto MP, Grou CP, Alencastre IS, Fransen M, Sa-Miranda C, Azevedo JE (2007b) Ubiquitination of mammalian Pex5p, the peroxisomal import receptor. J. Biol. Chem. 282 (43):31267-31272.

Chang CC, Warren DS, Sacksteder KA, Gould SJ (1999) PEX12 interacts with PEX5 and PEX10 and acts downstream of receptor docking in peroxisomal matrix protein import. J. Cell Biol. 147 (4):761-774.

Chang EJ, Ha J, Kang SS, Lee ZH, Kim HH (2011) AWP1 binds to tumor necrosis factor receptor-associated factor 2 (TRAF2) and is involved in TRAF2-mediated nuclear factor-kappaB signaling. Int. J. Biochem. Cell Biol. 43 (11):1612-1620.

Collins CS, Kalish JE, Morrell JC, McCaffery JM, Gould SJ (2000) The peroxisome biogenesis factors pex4p, pex22p, pex1p, and pex6p act in the terminal steps of peroxisomal matrix protein import. Mol. Cell. Biol. 20 (20):7516-7526.

Costa-Rodrigues J, Carvalho AF, Fransen M, Hambruch E, Schliebs W, Sa-Miranda C, Azevedo JE (2005) Pex5p, the peroxisomal cycling receptor, is a monomeric nonglobular protein. J. Biol. Chem. 280 (26):24404-24411.

Costa-Rodrigues J, Carvalho AF, Gouveia AM, Fransen M, Sa-Miranda C, Azevedo JE (2004) The $\mathrm{N}$ terminus of the peroxisomal cycling receptor, Pex5p, is required for redirecting the peroxisome-associated peroxin back to the cytosol. J. Biol. Chem. 279 (45):46573-46579.

Debelyy MO, Platta HW, Saffian D, Hensel A, Thoms S, Meyer HE, Warscheid B, Girzalsky W, Erdmann R (2011) Ubp15p, a ubiquitin hydrolase associated with the peroxisomal export machinery. J. Biol. Chem. 286 (32):28223-28234.

Deshaies RJ, Joazeiro CA (2009) RING domain E3 ubiquitin ligases. Annu. Rev. Biochem. 78:399-434.

Distel B, Erdmann R, Gould SJ, Blobel G, Crane DI, Cregg JM, Dodt G, Fujiki Y, Goodman JM, Just WW, Kiel JA, Kunau WH, Lazarow PB, Mannaerts GP, Moser HW, Osumi T, Rachubinski RA, Roscher A, Subramani S, Tabak HF, Tsukamoto T, 
Valle D, van der Klei I, van Veldhoven PP, Veenhuis M (1996) A unified nomenclature for peroxisome biogenesis factors. J. Cell Biol. 135 (1):1-3.

Dodt G, Braverman N, Wong C, Moser A, Moser HW, Watkins P, Valle D, Gould SJ (1995) Mutations in the PTS1 receptor gene, PXR1, define complementation group 2 of the peroxisome biogenesis disorders. Nat. Genet. 9 (2):115-125.

Dodt G, Gould SJ (1996) Multiple PEX genes are required for proper subcellular distribution and stability of Pex5p, the PTS1 receptor: evidence that PTS1 protein import is mediated by a cycling receptor. J. Cell Biol. 135 (6 Pt 2):1763-1774.

Dodt G, Warren D, Becker E, Rehling P, Gould SJ (2001) Domain mapping of human PEX5 reveals functional and structural similarities to Saccharomyces cerevisiae Pex18p and Pex21p. J. Biol. Chem. 276 (45):41769-41781.

Einwachter H, Sowinski S, Kunau WH, Schliebs W (2001) Yarrowia lipolytica Pex20p, Saccharomyces cerevisiae Pex18p/Pex21p and mammalian Pex5pL fulfil a common function in the early steps of the peroxisomal PTS2 import pathway. EMBO Rep. 2 (11):1035-1039.

Fransen M, Brees C, Baumgart E, Vanhooren JC, Baes M, Mannaerts GP, Van Veldhoven PP (1995) Identification and characterization of the putative human peroxisomal C-terminal targeting signal import receptor. J. Biol. Chem. 270 (13):7731-7736.

Fransen M, Nordgren M, Wang B, Apanasets $O$ (2012) Role of peroxisomes in ROS/RNS-metabolism: implications for human disease. Biochim. Biophys. Acta 1822 (9):1363-1373.

Freitas MO, Francisco T, Rodrigues TA, Alencastre IS, Pinto MP, Grou CP, Carvalho AF, Fransen M, Sa-Miranda C, Azevedo JE (2011) PEX5 protein binds monomeric catalase blocking its tetramerization and releases it upon binding the $\mathrm{N}$-terminal domain of PEX14. J. Biol. Chem. 286 (47):40509-40519.

Galland N, Demeure F, Hannaert V, Verplaetse E, Vertommen D, Van der Smissen P, Courtoy PJ, Michels PA (2007) Characterization of the role of the receptors PEX5 and PEX7 in the import of proteins into glycosomes of Trypanosoma brucei. Biochim. Biophys. Acta 1773 (4):521-535.

Galland N, Michels PA (2010) Comparison of the peroxisomal matrix protein import system of different organisms. Exploration of possibilities for developing inhibitors of the import system of trypanosomatids for anti-parasite chemotherapy. Eur. J. Cell Biol. 89 (9):621-637.

Gatto GJ, Jr., Geisbrecht BV, Gould SJ, Berg JM (2000) A proposed model for the PEX5peroxisomal targeting signal-1 recognition complex. Proteins 38 (3):241-246.

Gonen H, Bercovich B, Orian A, Carrano A, Takizawa C, Yamanaka K, Pagano M, Iwai K, Ciechanover A (1999) Identification of the ubiquitin carrier proteins, E2s, involved in signal-induced conjugation and subsequent degradation of IkappaBalpha. J. Biol. Chem. 274 (21):14823-14830.

Gould SJ, Keller GA, Hosken N, Wilkinson J, Subramani S (1989) A conserved tripeptide sorts proteins to peroxisomes. J. Cell Biol. 108 (5):1657-1664. 
Gouveia AM, Guimaraes CP, Oliveira ME, Reguenga C, Sa-Miranda C, Azevedo JE (2003a) Characterization of the peroxisomal cycling receptor, Pex5p, using a cell-free in vitro import system. J. Biol. Chem. 278 (1):226-232.

Gouveia AM, Guimaraes CP, Oliveira ME, Sa-Miranda C, Azevedo JE (2003b) Insertion of Pex5p into the peroxisomal membrane is cargo protein-dependent. J. Biol. Chem. 278 (7):4389-4392.

Gouveia AM, Reguenga C, Oliveira ME, Sa-Miranda C, Azevedo JE (2000) Characterization of peroxisomal Pex $5 p$ from rat liver. Pex $5 p$ in the Pex5p-Pex14p membrane complex is a transmembrane protein. J. Biol. Chem. 275 (42):3244432451.

Grou CP, Carvalho AF, Pinto MP, Alencastre IS, Rodrigues TA, Freitas MO, Francisco T, Sa-Miranda C, Azevedo JE (2009a) The peroxisomal protein import machinery--a case report of transient ubiquitination with a new flavor. Cell. Mol. Life Sci. 66 (2):254-262.

Grou CP, Carvalho AF, Pinto MP, Huybrechts SJ, Sa-Miranda C, Fransen M, Azevedo JE (2009b) Properties of the ubiquitin-pex5p thiol ester conjugate. J. Biol. Chem. 284 (16):10504-10513.

Grou CP, Carvalho AF, Pinto MP, Wiese S, Piechura H, Meyer HE, Warscheid B, SaMiranda C, Azevedo JE (2008) Members of the E2D (Ubch5) family mediate the ubiquitination of the conserved cysteine of Pex5p, the peroxisomal import receptor. J. Biol. Chem. 283 (21):14190-14197.

Grou CP, Francisco T, Rodrigues TA, Freitas MO, Pinto MP, Carvalho AF, Domingues P, Wood SA, Rodriguez-Borges JE, Sa-Miranda C, Fransen M, Azevedo JE (2012) Identification of ubiquitin-specific protease 9X (USP9X) as a deubiquitinase acting on ubiquitin-peroxin 5 (PEX5) thioester conjugate. J. Biol. Chem. 287 (16):12815-12827.

Gunkel K, van Dijk R, Veenhuis M, van der Klei IJ (2004) Routing of Hansenula polymorpha alcohol oxidase: an alternative peroxisomal protein-sorting machinery. Mol. Biol. Cell 15 (3):1347-1355.

Hensel A, Beck S, El Magraoui F, Platta HW, Girzalsky W, Erdmann R (2011) Cysteinedependent ubiquitination of Pex18p is linked to cargo translocation across the peroxisomal membrane. J. Biol. Chem. 286 (50):43495-43505.

Hu J, Baker A, Bartel B, Linka N, Mullen RT, Reumann S, Zolman BK (2012) Plant peroxisomes: biogenesis and function. Plant Cell 24 (6):2279-2303.

Hutchins AP, Liu S, Diez D, Miranda-Saavedra D (2013) The repertoires of ubiquitinating and deubiquitinating enzymes in eukaryotic genomes. Mol. Biol. Evol. 30 (5):11721187.

Islinger M, Cardoso MJ, Schrader M (2010) Be different--the diversity of peroxisomes in the animal kingdom. Biochim. Biophys. Acta 1803 (8):881-897.

Islinger M, Luers GH, Li KW, Loos M, Volkl A (2007) Rat liver peroxisomes after fibrate treatment. A survey using quantitative mass spectrometry. J. Biol. Chem. 282 (32):23055-23069. 
Joazeiro CA, Weissman AM (2000) RING finger proteins: mediators of ubiquitin ligase activity. Cell 102 (5):549-552.

Kaur N, Zhao Q, Xie Q, Hu J (2013) Arabidopsis RING peroxins are E3 ubiquitin ligases that interact with two homologous ubiquitin receptor proteins $(F)$. J. Integr. Plant Biol. $55(1): 108-120$.

Kiel JA, Emmrich K, Meyer HE, Kunau WH (2005a) Ubiquitination of the peroxisomal targeting signal type 1 receptor, Pex5p, suggests the presence of a quality control mechanism during peroxisomal matrix protein import. J. Biol. Chem. 280 (3):19211930.

Kiel JA, Otzen M, Veenhuis M, van der Klei IJ (2005b) Obstruction of polyubiquitination affects PTS1 peroxisomal matrix protein import. Biochim. Biophys. Acta 1745 (2):176-186.

Kiel JA, Veenhuis M, van der Klei IJ (2006) PEX genes in fungal genomes: common, rare or redundant. Traffic 7 (10):1291-1303.

Kikuchi M, Hatano N, Yokota S, Shimozawa N, Imanaka T, Taniguchi H (2004) Proteomic analysis of rat liver peroxisome: presence of peroxisome-specific isozyme of Lon protease. J. Biol. Chem. 279 (1):421-428.

Klein AT, Barnett P, Bottger G, Konings D, Tabak HF, Distel B (2001) Recognition of peroxisomal targeting signal type 1 by the import receptor Pex5p. J. Biol. Chem. 276 (18):15034-15041.

Klein AT, van den Berg M, Bottger G, Tabak HF, Distel B (2002) Saccharomyces cerevisiae acyl-CoA oxidase follows a novel, non-PTS1, import pathway into peroxisomes that is dependent on Pex5p. J. Biol. Chem. 277 (28):25011-25019.

Koller A, Snyder WB, Faber KN, Wenzel TJ, Rangell L, Keller GA, Subramani S (1999) Pex22p of Pichia pastoris, essential for peroxisomal matrix protein import, anchors the ubiquitin-conjugating enzyme, Pex4p, on the peroxisomal membrane. J. Cell Biol. $146(1): 99-112$.

Kragt A, Voorn-Brouwer T, van den Berg M, Distel B (2005) The Saccharomyces cerevisiae peroxisomal import receptor Pex5p is monoubiquitinated in wild type cells. J. Biol. Chem. 280 (9):7867-7874.

Kunze M, Neuberger G, Maurer-Stroh S, Ma J, Eck T, Braverman N, Schmid JA, Eisenhaber F, Berger J (2011) Structural requirements for interaction of peroxisomal targeting signal 2 and its receptor PEX7. J. Biol. Chem. 286 (52):45048-45062.

Kurochkin IV, Mizuno Y, Konagaya A, Sakaki Y, Schonbach C, Okazaki Y (2007) Novel peroxisomal protease Tysnd1 processes PTS1- and PTS2-containing enzymes involved in beta-oxidation of fatty acids. EMBO J. 26 (3):835-845.

Lazarow PB (2006) The import receptor Pex7p and the PTS2 targeting sequence. Biochim. Biophys. Acta 1763 (12):1599-1604.

Leon S, Subramani S (2007) A conserved cysteine residue of Pichia pastoris Pex20p is essential for its recycling from the peroxisome to the cytosol. J. Biol. Chem. 282 (10):7424-7430. 
Leon S, Zhang L, McDonald WH, Yates J, 3rd, Cregg JM, Subramani S (2006) Dynamics of the peroxisomal import cycle of PpPex20p: ubiquitin-dependent localization and regulation. J. Cell Biol. 172 (1):67-78.

Liu X, Subramani S (2013) Unique requirements for mono- and polyubiquitination of the peroxisomal targeting signal co-receptor, Pex20. J. Biol. Chem. 288 (10):7230-7240.

Ma C, Subramani S (2009) Peroxisome matrix and membrane protein biogenesis. IUBMB Life 61 (7):713-722.

Matsumoto N, Tamura S, Fujiki Y (2003) The pathogenic peroxin Pex26p recruits the Pex1p-Pex6p AAA ATPase complexes to peroxisomes. Nat. Cell Biol. 5 (5):454-460.

Metzger MB, Hristova VA, Weissman AM (2012) HECT and RING finger families of E3 ubiquitin ligases at a glance. J. Cell Sci. 125 (Pt 3):531-537.

Metzger MB, Pruneda JN, Klevit RE, Weissman AM (2013) RING-type E3 ligases: Master manipulators of E2 ubiquitin-conjugating enzymes and ubiquitination. Biochim. Biophys. Acta. doi:10.1016/j.bbamcr.2013.05.026.

Michels PA, Bringaud F, Herman M, Hannaert V (2006) Metabolic functions of glycosomes in trypanosomatids. Biochim. Biophys. Acta 1763 (12):1463-1477.

Miyata N, Okumoto K, Mukai S, Noguchi M, Fujiki Y (2012) AWP1/ZFAND6 functions in Pex5 export by interacting with cys-monoubiquitinated Pex5 and Pex6 AAA ATPase. Traffic 13 (1):168-183.

Oeljeklaus S, Reinartz BS, Wolf J, Wiese S, Tonillo J, Podwojski K, Kuhlmann K, Stephan C, Meyer HE, Schliebs W, Brocard C, Erdmann R, Warscheid B (2012) Identification of core components and transient interactors of the peroxisomal importomer by dual-track stable isotope labeling with amino acids in cell culture analysis. J. Proteome Res. 11 (4):2567-2580.

Oliveira ME, Gouveia AM, Pinto RA, Sa-Miranda C, Azevedo JE (2003) The energetics of Pex5p-mediated peroxisomal protein import. J. Biol. Chem. 278 (41):39483-39488.

Oshima Y, Kamigaki A, Nakamori C, Mano S, Hayashi M, Nishimura M, Esaka M (2008) Plant catalase is imported into peroxisomes by Pex5p but is distinct from typical PTS1 import. Plant Cell Physiol. 49 (4):671-677.

Otera H, Harano T, Honsho M, Ghaedi K, Mukai S, Tanaka A, Kawai A, Shimizu N, Fujiki $Y$ (2000) The mammalian peroxin Pex5pL, the longer isoform of the mobile peroxisome targeting signal (PTS) type 1 transporter, translocates the Pex7p.PTS2 protein complex into peroxisomes via its initial docking site, Pex14p. J. Biol. Chem. 275 (28):21703-21714.

Otera H, Okumoto K, Tateishi K, Ikoma Y, Matsuda E, Nishimura M, Tsukamoto T, Osumi T, Ohashi K, Higuchi O, Fujiki Y (1998) Peroxisome targeting signal type 1 (PTS1) receptor is involved in import of both PTS1 and PTS2: studies with PEX5defective $\mathrm{CHO}$ cell mutants. Mol. Cell. Biol. 18 (1):388-399.

Platta HW, El Magraoui F, Baumer BE, Schlee D, Girzalsky W, Erdmann R (2009) Pex2 and pex12 function as protein-ubiquitin ligases in peroxisomal protein import. Mol. Cell. Biol. 29 (20):5505-5516. 
Platta HW, El Magraoui F, Schlee D, Grunau S, Girzalsky W, Erdmann R (2007) Ubiquitination of the peroxisomal import receptor Pex5p is required for its recycling. J. Cell Biol. 177 (2):197-204.

Platta HW, Girzalsky W, Erdmann R (2004) Ubiquitination of the peroxisomal import receptor Pex5p. Biochem J. 384 (Pt 1):37-45.

Platta HW, Hagen S, Erdmann R (2013) The exportomer: the peroxisomal receptor export machinery. Cell. Mol. Life Sci. 70 (8):1393-1411.

Reguenga C, Oliveira ME, Gouveia AM, Sa-Miranda C, Azevedo JE (2001) Characterization of the mammalian peroxisomal import machinery: Pex $2 p$, Pex $5 p$, Pex12p, and Pex14p are subunits of the same protein assembly. J. Biol. Chem. 276 (32):29935-29942.

Rokka A, Antonenkov VD, Soininen R, Immonen HL, Pirila PL, Bergmann U, Sormunen RT, Weckstrom M, Benz R, Hiltunen JK (2009) Pxmp2 is a channel-forming protein in Mammalian peroxisomal membrane. PLoS One 4 (4):e5090.

Saidowsky J, Dodt G, Kirchberg K, Wegner A, Nastainczyk W, Kunau WH, Schliebs W (2001) The di-aromatic pentapeptide repeats of the human peroxisome import receptor PEX5 are separate high affinity binding sites for the peroxisomal membrane protein PEX14. J. Biol. Chem. 276 (37):34524-34529.

Sarikas A, Hartmann T, Pan ZQ (2011) The cullin protein family. Genome Biol. 12 (4):220.

Saville MK, Sparks A, Xirodimas DP, Wardrop J, Stevenson LF, Bourdon JC, Woods YL, Lane DP (2004) Regulation of p53 by the ubiquitin-conjugating enzymes UbcH5B/C in vivo. J. Biol. Chem. 279 (40):42169-42181.

Schliebs W, Kunau WH (2006) PTS2 co-receptors: diverse proteins with common features. Biochim. Biophys. Acta 1763 (12):1605-1612.

Schluter A, Fourcade S, Ripp R, Mandel JL, Poch O, Pujol A (2006) The evolutionary origin of peroxisomes: an ER-peroxisome connection. Mol. Biol. Evol. 23 (4):838-845.

Schuhmann H, Huesgen PF, Gietl C, Adamska I (2008) The DEG15 serine protease cleaves peroxisomal targeting signal 2-containing proteins in Arabidopsis. Plant Physiol. 148 (4):1847-1856.

Shiozawa K, Konarev PV, Neufeld C, Wilmanns M, Svergun DI (2009) Solution structure of human Pex5.Pex14.PTS1 protein complexes obtained by small angle X-ray scattering. J. Biol. Chem. 284 (37):25334-25342.

Spasser L, Brik A (2012) Chemistry and biology of the ubiquitin signal. Angew. Chem. Int. Ed. Engl. 51 (28):6840-6862.

Swinkels BW, Gould SJ, Bodnar AG, Rachubinski RA, Subramani S (1991) A novel, cleavable peroxisomal targeting signal at the amino-terminus of the rat 3-ketoacylCoA thiolase. EMBO J. 10 (11):3255-3262.

Wanders RJ, Waterham HR (2006) Biochemistry of mammalian peroxisomes revisited. Annu. Rev. Biochem. 75:295-332. 
Waterham HR, Ebberink MS (2012) Genetics and molecular basis of human peroxisome biogenesis disorders. Biochim. Biophys. Acta 1822 (9):1430-1441.

Wiebel FF, Kunau WH (1992) The Pas2 protein essential for peroxisome biogenesis is related to ubiquitin-conjugating enzymes. Nature 359 (6390):73-76.

Wiese S, Gronemeyer T, Ofman R, Kunze M, Grou CP, Almeida JA, Eisenacher M, Stephan C, Hayen $H$, Schollenberger L, Korosec T, Waterham HR, Schliebs W, Erdmann R, Berger J, Meyer HE, Just W, Azevedo JE, Wanders RJ, Warscheid B (2007) Proteomics characterization of mouse kidney peroxisomes by tandem mass spectrometry and protein correlation profiling. Mol. Cell. Proteomics 6 (12):2045-2057.

Williams C, van den Berg M, Geers E, Distel B (2008) Pex10p functions as an E3 ligase for the Ubc4p-dependent ubiquitination of Pex5p. Biochem. Biophys. Res. Commun. $374(4): 620-624$.

Williams C, van den Berg M, Sprenger RR, Distel B (2007) A conserved cysteine is essential for Pex4p-dependent ubiquitination of the peroxisomal import receptor Pex5p. J. Biol. Chem. 282 (31):22534-22543.

Woodward AW, Bartel B (2005) The Arabidopsis peroxisomal targeting signal type 2 receptor PEX7 is necessary for peroxisome function and dependent on PEX5. Mol. Biol. Cell 16 (2):573-583.

Yahraus T, Braverman N, Dodt G, Kalish JE, Morrell JC, Moser HW, Valle D, Gould SJ (1996) The peroxisome biogenesis disorder group 4 gene, PXAAA1, encodes a cytoplasmic ATPase required for stability of the PTS1 receptor. EMBO J. 15 (12):2914-2923.

Zolman BK, Monroe-Augustus M, Silva ID, Bartel B (2005) Identification and functional characterization of Arabidopsis PEROXIN4 and the interacting protein PEROXIN22. Plant Cell 17 (12):3422-3435. 


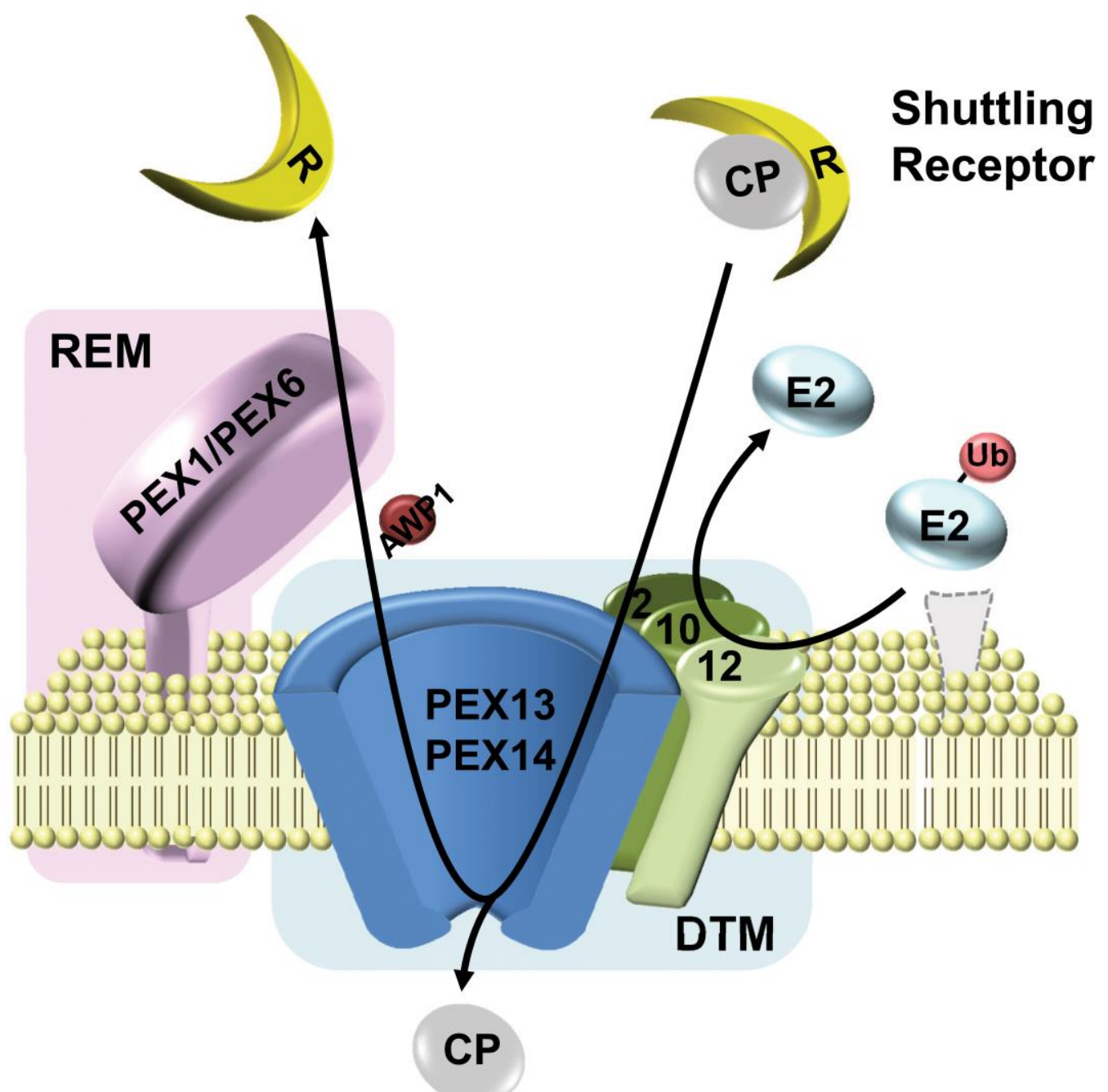

Fig. 4.4.1 

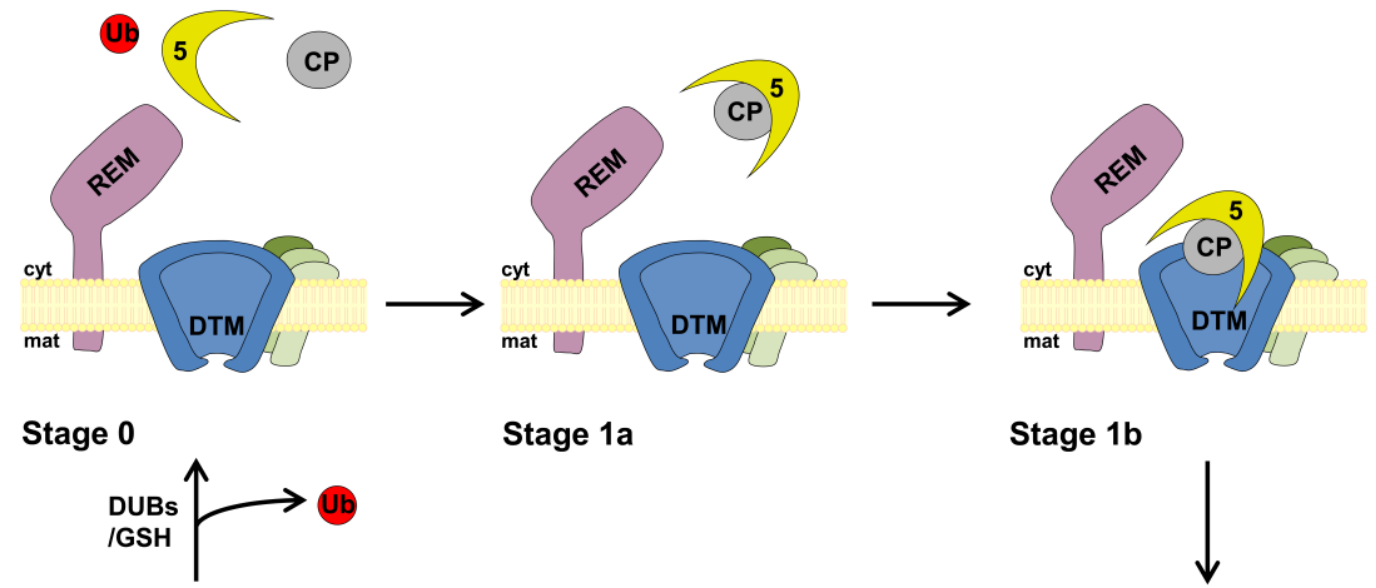

Stage 1a

Stage 1b

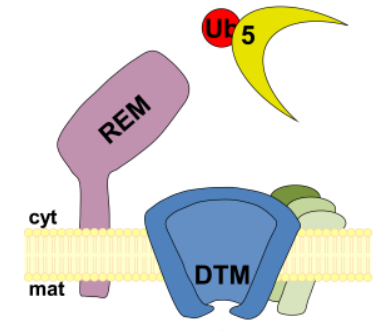

Stage 4b CP
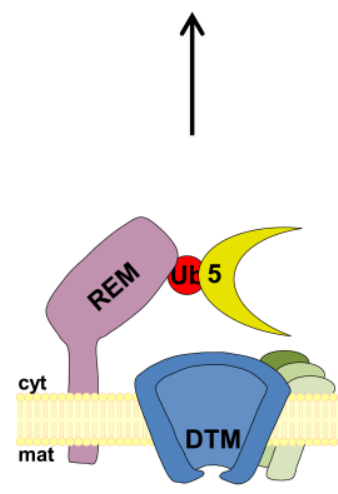

Stage 4a CP

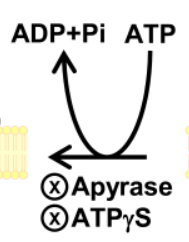

囚ATP $\gamma$

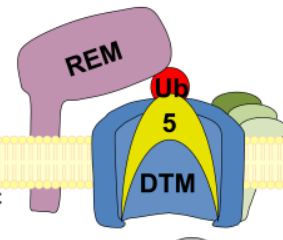

Stage 3b

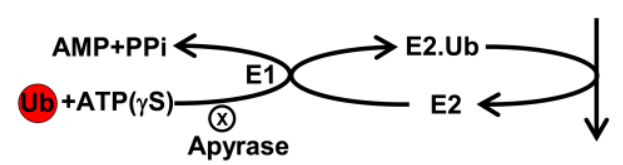

Stage 2 CP
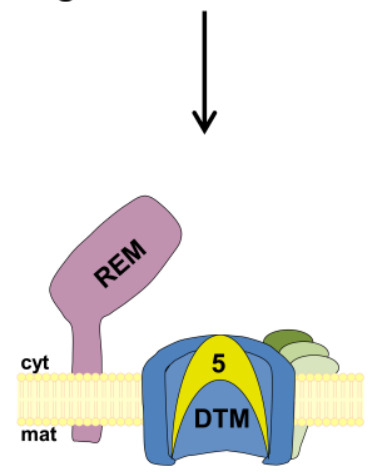